\title{
The influence of long-term practice on mental rotation of 3-D objects
}

\author{
Gilles Leone *, Marie Claire Taine, Jacques Droulez \\ Laboratoire de Physiologie de la Perception et de l'Action, C.N.R.S, Collège de France, 15 rue de l'Ecole de Médecine, Paris, France
}

(Accepted 16 December 1993)

Key words: Mental rotation; 3-D object; Practice: Imaging skill; Complexity

\begin{abstract}
We eviluated the influence of long-term practice on the performance of a mental rotation task in which subjects judged whether two 3-D objects presented in different orientations were identical. Stimuli and experimental conditions were analogous to those used by Shepard and Metzler. Sixtcen subjects were selected, to test the influence of aptitude for mental imagery on this learning process. Subjects participed in 12 to 15 sessions over 6 weeks. Two catalogues of different stimuli were alternatively used during three (or six) consecutive sessions to determine the influence of complexity and familiarity of figures. For all subjects, the inverse of the velocity of mental rotation along the sessions was adequately fitted by a decreasing exponential curve. However, evidence for mental rotation did not disappear, even after 15 sessions. Asymptotic variations can be attributed to differences in stimuli as well as imaging skills of subjects. Our results lead to a new interpretation of the mental rotation proces:
\end{abstract}

\section{INTRODUCTION}

The paradigm of mental rotation, as introduced by Shepard and Metzler ${ }^{27}$, gives insight into the processes of image representations and manipulations. This is a paradigm in which subjects were asked to determine whether two figures displayed in different orientations were identical or not. Subjects were simultaneously shown pairs of images with different orientations, which had been constructed from five rigid 3-D structures and their enantiomorphs (mirror-reflected images). The subjects' response times (RT) were linearly related to the difference in orientation (OD), giving support to the hypothesis that they mentally rotate the stimulus in this identification process.

Sirce then, many experiments of this type have been performed under a variety of conditions (i.e. different stimuli, different modes of presentation) leading to specific RT-OD functions that often reproduce this linearity. Nevertheless, the experiments concerned with specific influence of practice on this mental rotation process have produced some conflicting results.

In the initial experiment by Shepard and Metzler ${ }^{27}$, subjects were presented with 320 pairs of images in one continuous session. In a study of practice effect, Metz-

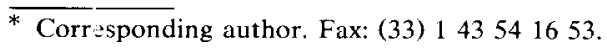

ler ${ }^{21}$, using a single 3-D object (and its enantiomorph) found a constant rate of mental rotation over 1728 trials. On the other hand, with seven blocks of 72 trials (with no rest period between consecutive blocks) using two basic objects, Kaushall and Parsons ${ }^{16}$ obtained response times which were unrelated to the difference in orientation by the time the seventh block was reached. This suggests that subjects no longer used mental rotation after practice. In the latter experiment, the two shapes were successively presented, the first one always corresponded to a 'standard object' in a constant orientation. Subjects were informed of this fact but not of the strategy to be used. The introduction of a new but similar object within the fifth block had no effect on the slope of the RT-OD function. The authors, however, gave no description of the difference between the new and old objects.

Using 2-D matrices of black and white squares, Bethell-Fox and Shepard ${ }^{3}$ observed response times that remained dependent on the rotation angle with practice but became independent of the complexity of stimuli, as defined by the 'number of perceptually distinct pieces' in their $3 \times 3$ grid stimuli. The introduction of new shapes showed a mental rotation rate that was complexity dependent, but this effect also disappeared with time. These variable results may bc explained by different experimental conditions such as the type (nature, familiarity, complexity) of stimuli, 
their number, or their presentation mode. This latter consideration can lead to possible memorisation of the stimuli and reduction in the need for mental rotation ${ }^{30}$. According to Steiger and Yuille ${ }^{30}$, the complexity dependence of the mental rotation process was a key argument for rejecting Shepard and Metzler's hypothe$\operatorname{sis}^{27}$.

Yet, none of these previous experiments were based on long-term training with an appropriate number of stimuli that would impede an easy memorisation.

We investigated this effect of practice on mental rotation in a set of experiments similar to that carried out by Shepard and Metzler ${ }^{27}$. We used identical or mirror-reflected pairs of 3-D objects which were simultaneously and randomly presented to subjects. Our purpose was to study: (i) if there was an influence of practice, and if so, (ii) the characteristics of this training (the evolution of the parameters of the RT-OD function), (iii) the stimulus dependence on this practice, and since these stimuli were grouped in two sets, the set dependence on this practice.

A fundamental feature of our experiment is that our procedure could hardly allow a direct memorisation of stimuli.

In addition to issues of practice, differences between individual aptitudes for performing mental rotation have been reported. These differences may be attributed to general abilities in spatial viewing and to differing processing strategies ${ }^{14,20}$. A differential approach to human image processing based on moduli of mental image treatment was proposed by Kosslyn ${ }^{17}$ and refined by Kosslyn and Jolicoeur ${ }^{19}$ and Schwartz and Kosslyn ${ }^{25}$. These authors suggest that the manipulation of mental images requires, for example, firstly the activation of the image in a visual buffer (called 'picture' modulus by the authors), then its retention in this visual buffer ('regenerate' modulus), its mental scanning ('scan' modulus) or its mental rotation ('rotate' modulus). The performance of a subject in a task involving mental imagery will depend on performance related to each modulus. Denis ${ }^{9}$, giving support to this theory, has recently provided a definition of 'good imagers' as those who possess the following characteristics: (i) specific abilities in the functionally independent moduli of human image processing, (ii) the ability to identify situations in which imagery seems to be useful, and (iii) a preference for using mental imagery to solve certain tasks.

Tapley and Briden ${ }^{32}$ noted a gender difference resulting in lower performance for mental rotation for female subjects. Richardson ${ }^{24}$, however, showed that this difference disappears as the educational level of the subject increases. We tested whether differences in

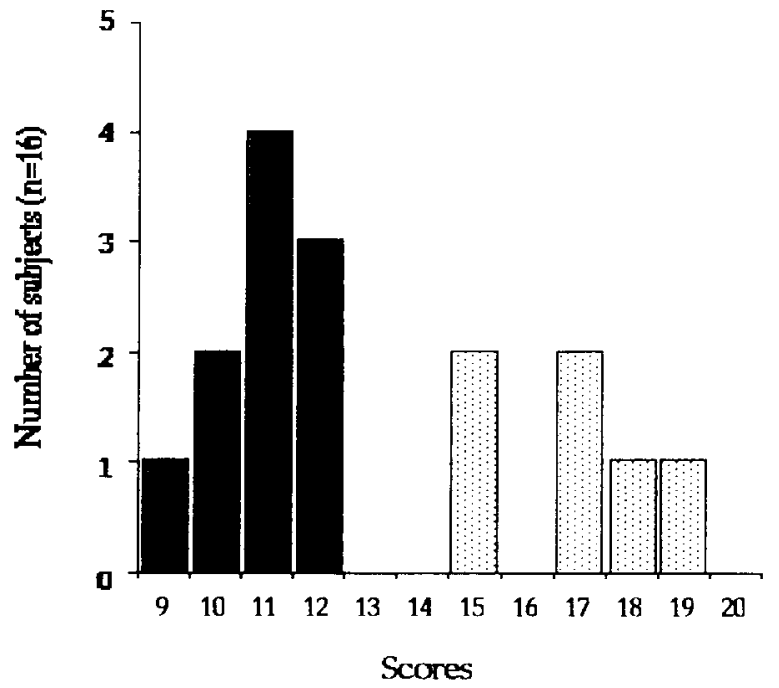

\footnotetext{
"Pocri" Imagers

"Good" Irmagere

(Mean $\pm 5 \Gamma ; 10.9 \pm 0.95)$

(Mean $\pm 5 D$ ) $16.8 \pm 1.5$ )
}

Fig. 1. Classification of subjects according to their scores for the paper Mental Rotation Test. The subjects can be divided into two groups. Subjects with high scores, more than 14 , are classified as 'good' imagers and subjects with scores lower than 12 are classified as 'poor' imagers.

the performance during practice of mental rotation between 'poor imagers' and 'good imagers' reflect underlying differences in imaging skills.

A preliminary account of the results was previously presented $^{31}$.

\section{MATERIALS AND METHODS}

Subjects

Twenty subjects ( 10 male, 10 female) were designated as ('good imagers' or 'poor imagers') on the basis of their scores for the paper Mental Rotation Test ${ }^{33}$. Most were students between the ages of 20 and 25 , and they were divided into four groups. Each group contained subjects of both sexes and both imaging abilities. Each group had at least one subject of a determined profile (sex, ability) for comparison purposes and was submitted to different experimental designs as explained below. Four females decided against continuing the experiment resulting in a modification of our initial objectives. The results presented here are therefore for 16 subjects (10 male and 6 female) without reference to gender differences (see Fig. 1). The training consisted on average of two regular sessions per week. Subjects were paid for each session.

Stimuli

Stimuli consisted of perspective views of 3-D wire frame rigid objects composed of ten contiguous cubes in different spatial orientations. These views were generated by computer software. The lines composing the views were antialiased by software (i.e., a gaussian filtering of intensity was applied to the lines in order to eliminate staircase effect due to pixel sampling of the screen), and had a width of $1 \mathrm{~mm}$. All lines of the cubes were drawn in grey scale (the uniform white has a luminance of about $80 \mathrm{~cd} / \mathrm{m}^{2}$ ), against the dark background of the screen (with a luminance of about $0.04 \mathrm{~cd} / \mathrm{m}^{2}$ ). The mean luminance of a perspective view of an object was $5.5 \mathrm{~cd} / \mathrm{m}^{2}$ at $60 \mathrm{~cm}$ from the screen. The computer screen on which the figures were displayed had $800 \times 600$ pixel resolution.

Two series of stimuli were used (catalogues $A$ and B); each containing 156 examples consisting of the simultaneous presentation of two views. For each catalogue, these two views were obtained with 
CATALOGA
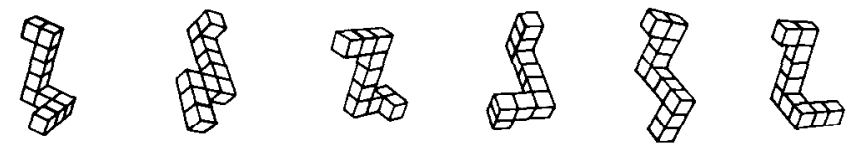

CATALOG B
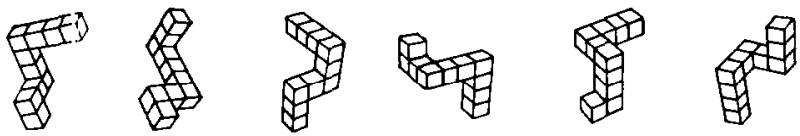

Fig. 2. Single view of the twelve objects used in this experiment. These ubjects were equally and randomly divided in two catalogues $A$ and $B$.

six objects and their enantiomorphs which were displayed in different or entations (rotations in 'depth' or in the 'picture-plane' between $l^{\circ}$ and $180^{\circ}$ in multiples of $30^{\circ}$ ). The orientation of each pair of vieus was random but selected so as to avoid occluded or ambiguous parts. Fig. 2 shows a single view of each of the 6 objects in each of the two catalogues.

\section{Proced:re}

Suljects were seated in a dark room with the screen at a distance of $60 \mathrm{~cm}$ from their eyes. The screen was viewed through a cylindrical black optical tube to avoid any external reference frames. Each image covered approximately $8^{\circ}$ of visual arc in height and width and was seen binocularly.

Subjects pressed the right button to indicate that the two objects were iclentical or the left button to indicate a mirror-reflected pair. Each txperimental session lasted $30 \mathrm{~min}$ with 10 -s pauses every 4 min. The subject's response triggered the appearance of the next pair. The presentation immediately followed an 'attention' message and did not exceed $15 \mathrm{~s}$ : if this time was not sufficient, the stimulus was removed, the trial was rejected and instructions were delivered on the screen to continuc. This time was sufficiently long for virtually all responses. Only one catalogue was used for each session, and trials were randomly presented over $30 \mathrm{~min}$. Their number depended on the subject's speed, but each object was approximately equally presented. Sessions were mixed with 'picture-plane' and 'depth' rotations.

The entire system was controlled by a MOTOROLA 68030 microprocessor and a graphic QPDM coprocessor. The operating system OS9 recorded response times with a resolution of $80 \mu \mathrm{s}$.

Subiects were asked to use mental rotation to determine the congruence of the two views and were not informed of the change of catalogue (if any) or specific rotation axis in advance. The strategy of mental rotation was first introduced to subjects in the paper test in which hey were asked to picture and to rotate a mental figure in their mind as they would have done with a physical object.

They were, however, informed of the following: (i) the different cases that could occur ('picture-plane' or 'depth' rotations with objects that were identical or enantiomorphic); (ii) the sole consideration of the first button press; (iii) the random orientation of the pairs; (iv) the random occurrence of the trial; and (v) the length of session, and pauses.

\section{Experimental design}

Each group performed a certain sequence of the two catalogues ( $\mathrm{A}$ and $\mathrm{B}$ ): $3 \mathrm{~A} / 3 \mathrm{~B} / 3 \mathrm{~A} / 3 \mathrm{~B}, 6 \mathrm{~A} / 6 \mathrm{~B} / 3 \mathrm{~A}$ and symmetries, $3 \mathrm{~B} / 3 \mathrm{~A}$ / $3 \mathrm{~B} / 3 \mathrm{~A}$ and $6 \mathrm{~B} / 6 \mathrm{~A} / 3 \mathrm{~B}$. We wished to analyse the transition effect from one catalogue to the other as well as long-term practice effect across catalogues.

\section{RESULTS}

The sixteen subjects performed all the sessions, according to their group (12 or 15 sessions), however, data for six sessions (four with catalogue $A$ and two with catalogue B), collected on five subjects, were impossible to analyse due to technical problems and were considered as missing values in further statistical analyses. Initially, we treated separately the results obtained for the two catalogues. In the subsequent analyses of dependent variables (error rate, coefficient of correlation, intercept and slopes of the regression lines), first we report the effects due to intrinsic properties of the stimuli (difference between catalogues, between axes of rotation or between enantiomorphic and identical stimuli) for all subjects and over all sessions. Second, we report the effects due to imaging abilities and number of sessions. Finally, we report the effects due to change of catalogues and novelty of stimuli.

\section{Effects of intrinsic properties of the stimuli}

In this part, we will demonstrate that subjects gave more incorrect responses, as well as rotated mentally more slowly, the stimuli from catalogue B than those from catalogue $\mathrm{A}$.

\section{Error rate}

We tested whether there are differences in the error rate due to catalogues or to the nature of stimuli. For each subject and each session, we computed the percentage of incorrect responses, i.e. the 'error rate'. We divided data according to the identical or enantiomorphic nature of stimuli. As these numbers were relatively small (less than 20\%), we made the statistical analyses after adding 1 to these numbers and then taking their square roots ${ }^{29}$. We call the resulting value the transformed error.

The overall mean (for all subjects over all sessions) of the transformed error was $1.66 \pm 0.85$ (mean \pm S.D.) and $1.84 \pm 0.95$ (mean \pm S.D.) for catalogue A (identical and enantiomorphic stimuli, respectively), and 2.30 $\pm 0.95($ mean \pm S.D. $)$ and $2.60 \pm 1.05$ (mean \pm S.D. $)$ for catalogue B. Subjects gave more incorrect responses on catalogue B than on catalogue A for both identical and enantiomorphic stimuli (one tailed $t$-test: $t_{203}=-5.29, P<0.0001$ for identical stimuli and $t_{203}$ $=-5.49, P<0.0001$ for enantiomorphic stimuli).

Subjects tended to give more incorrect responses for enantiomorphic stimuli than for identical stimuli for both catalogues. This effect was significant for catalogue B (one-tailed $t$-test: $t_{202}=-1.96, P<0.05$ ), but 
did not reach a significant level for catalogue A $(P>$ $0.10)$. The low percentage of incorrect responses on both catalogues showed that subjects clearly understood the task.

\section{Analyses of response time ( $R T)$}

For subsequent analysis, we considered only the correct responses of subjects for trials in which the objects were identical. Moreover, we divided data according to the axis of rotation. For each subject and each session, we performed a linear regression analysis on raw response times (RT), as a function of the difference of orientation between the two figures (OD). These linear regressions gave us three parameters: (i) the intercept, which represents the time required by a subject to perform the discrimination task when two figures are in the same orientation, predicted by linear model; (ii) the coefficient of correlation, which indicates the quality of fit of the linear model; and (iii) the slope of the regression line. The inverse of slope corresponds to the rate of the mental transformation.

\section{A-Coefficient of correlation}

Prior to doing statistical analysis on these values, we transformed them according to the Z-transformation used in Snedecor and Cochran ${ }^{28}$, in order to get rid of the skewed distribution of coefficient of correlation. This transformation of the coefficient of correlation is called the 'transformed coefficient' in the following.

The mean values (mean \pm S.D.) of the transformed coefficient were $0.73+0.19$ and $0.82 \pm 0.17$ for the picture-plane' rotation (for catalogue $\mathrm{A}$ and $\mathrm{B}$, respectively), $0.70 \pm 0.17$ and $0.78 \pm 0.2$ for 'depth' rotation (for catalogue $\mathrm{A}$ and $\mathrm{B}$, respectively).

The transformed coefficient was always higher for catalogue B than for catalogue A (one-tailed $t$-test; $t_{2013}=-3.79, P<0.001$ and $t_{203}=-3.01, P<0.005$ for 'picture-plane' and 'depth' rotation, respectively).

We can also notice that the transformed coefficient was usually higher for 'picture-plane' than for 'depth' rotation (paired $t$-test; $t_{102}=2.17, P<0.05$ for catalogue $\mathrm{A}$ and $t_{101}=2.55, P<0.05$ for catalogue $\mathrm{B}$ ).

In summary, we found that the linear model was adequate to describe the data. Moreover, the quality of the fit is dependent on the catalogue and on the axis of rotation.

\section{$B$ - Intercept}

We tested whether differences between the catalogues and the axes of rotation reported above for the coefficient of correlation exist also for the intercept. The mean values of the intercept were $1.32 \pm 1.04 \mathrm{~s}$ (mean \pm S.D.) and $1.07 \pm 0.59 \mathrm{~s}$ for 'picture-plane' ro- tation (for catalogues A and B, respectively), $1.26 \pm 0.75$ $\mathrm{s}$ and $1.41 \pm 0.72 \mathrm{~s}$ for 'depth' rotation (for catalogues $A$ and $B$, respectively).

The values of intercept were generally not significantly different for catalogue B and catalogue A (twotailed $t$-test; $t_{20,5}=2.05, P>0.04$ and $t_{20.5}=-1.40$, $P>0.15$ for 'picture-plane' and 'depth' rotation, respectively). Similarly, the values of intercept were not significantly different for 'picture-plane' and 'depth' rotations for catalogue $\mathrm{A}$ (paired $t$-test; $t_{102}=-0.3$, $P>0.75$ ), but were significantly different for catalogue B (paired $t$-test; $t_{101}=-7.1, P<0.001$ ).

In summary, there were no significant differences between the two catalogues regardless of the time required to perform a judgement of equality, when the two figures had no difference in orientation.

\section{$C$ - Slope of the regression lines}

Finally, we tested whether the slopes of the regression lines were dependent on the catalogues and/or on the axes of rotation. The mean value of the slope was $1.17 \pm 0.68 \mathrm{~ms} / \mathrm{deg}$ and $2.11 \pm 0.92 \mathrm{~ms} / \mathrm{deg}$ (mean \pm S.D.) for 'picture-plane' rotation (for catalogue $A$ and $\mathrm{B}$, respectively), $1.30 \pm 0.75 \mathrm{~ms} / \mathrm{deg}$ and $1.90 \pm 0.87$ $\mathrm{ms} / \mathrm{deg}$ for 'depth' rotation (for catalogue $\mathrm{A}$ and $\mathrm{B}$, respectively). The inverse of slope corresponds to the 'velocity' of the mental transformation. The mean 'velocities' were, respectively for catalogues A and B, $85 \mathrm{deg} / \mathrm{s}$ and $47 \mathrm{deg} / \mathrm{s}$ for 'picture-plane' rotation, 76 $\mathrm{deg} / \mathrm{s}$ and $52 \mathrm{deg} / \mathrm{s}$ for 'depth' rotation. These values were in the same range as those reported in previous studies using the same paradigm and similar 3-D objects ${ }^{27}$.

In contrast with intercept values, the values of slopes were significantly different for catalogue $B$ and catalogue A (one-tailed $t$-test; $t_{205}=-8.24, \quad P<0.0001$ and $t_{205}=-5.26, P<0.0001$ for 'picture-plane' and 'depth' rotation, respectively).

We noted that the value of slope for 'picture-plane' rotation does not differ from that for 'depth' rotation, for either catalogue A (two-tailed $t$-test; $t_{204}=1.26$, $P>0.20$ ), or catalogue B (two-tailed $t$-test; $t_{2012}=$ $-1.62, P>0.10)$.

In summary, we can point out that the rate of mental rotation is lower for catalogue $B$ than for catalogue $\mathrm{A}$, but no difference can be attributed to axes of rotation.

\section{Effects of practice and individual imaging ability}

In this part, we tested the effect of learning and mental imagery skill by performing an analysis of variance (ANOVA) on the parameters defined above 
(transformed error, transformed coefficient, intercept, slope), with the number of sessions and the imager class ('good' or 'poor' as defined previously) as factors. For each of those parameters, we will indicate the effect of each factor alone (session number or imaging ability) as well as the interaction betwcen them.

We will demonstrate that the significant differences in performance due to imaging skills or due to catalogues remain more or less constant over the training period. We will also point out that a simple exponential decreasing model can help describe the data for the slope (i.e. the inverse of mental rotation) over the training period.

\section{Error rate}

Imaging skill. The factor 'imager class' was highly significant for both catalogues and for both identical and enantiomorphic stimuli on the transformed error ( $F_{1,73}=8.7, P<0.01$ (identical stimuli), $F_{1,73}=6.9, P$ $<0.05$ (enantiomorphic stimuli) for catalogue $\mathrm{A}$ and $F_{1,72}=8.0, P<0.01$ (identical stimuli), $F_{1,72}=24.8, P$ $<0.0001$ (enantiomorphic stimuli) for catalogue B). Indeed, 'good' imagers made fewer errors on both catalogues over the entire training period (mean values of error rate: $1.0 \%$ (identical stimuli) and $1.70 \%$ (enantiomorphic stimuli) for catalogue $\mathrm{A}$ and $3.0 \%$ (identical stimuli) and $3.20 \%$ (enantiomorphic stimuli) for catalogue B) than 'poor' imagers (mean values of error rate: $3.6 \%$ (identical stimuli) and $4.50 \%$ (enantiomorphic stimuli) for catalogue $A$ and $6.70 \%$ (identical stimuli) and $9.00 \%$ (enantiomorphic stimuli) for catalogue B).

Effect of training. The mean error rate tended to decrease over the training period, but the change was not significant for either catalogue or for identical or enantiomorphic stimuli (the four $F$-tests: $P>0.1$ ).

Imaging skill/training cross effect. There was no significant interaction between the factor imager class and the session number for either catalogue or either nature of stimuli (identical or enantiomorphic) (the four $F$-test: $P>0.75$ ).
In summary, for initial trials, there is a difference in the accuracy of the discrimination between 'good' and 'poor' imagers. Although the rate of errors decreased for both classes, they decreased in parallel such that the initial difference between those groups persisted throughout the experimental period.

\section{Analyses of response time (RT)}

We considered the effect of training and imaging skill on coefficient of correlation, intercept and slope. As above, we studied only the correct responses of subjects for trials in which the objects were identical, and we divided data according to the axis of rotation.

\section{A - Coefficient of correlation}

Neither factor alone (imaging skill, session number) has a significant effect. However, there was in general a significant interaction between the two factors, imaging skill $\times$ session number, (but only marginally significant for 'depth' rotation with catalogue A). This suggests a differential influence of practice on 'poor' versus 'good' imagers. The values of the F-tests are given in Table $\mathbf{I}$.

'Poor' imagers and practice. An ANOVA performed on the subgroup of 'poor' imagers showed that there was generally no significant effect of number of sessions on the transformed coefficient $\left(F_{14,50}=1.18, P>\right.$ 0.3 for catalogue $\mathrm{B}$ and 'picture-plane' rotation, $F_{14,50}$ $=1.0, P>0.45$ for catalogue $\mathrm{B}$ and 'depth' rotation, $F_{14,43}=2.0, P>0.03$ for catalogue $\mathrm{A}$ and 'pictureplane' rotation and finally $F_{14,43}=0.498, P>0.9$ for catalogue $\mathrm{A}$ and 'depth' rotation).

The correlation coefficient did not change over the training period for 'poor' imagers.

'Good' imagers and practice. For 'good' imagers, there was generally a significant effect of session number on the transformed coefficient $\left(F_{14.23}=3.6, P<\right.$ 0.005 for catalogue B and 'picture-plane' rotation, $F_{14,23}=3.6, P<0.005$ for catalogue B and 'depth' rotation and $F_{14.30}=3.5, P<0.005$ for catalogue $\mathrm{A}$ and 'depth' rotation), except for catalogue $A$ and 'picture-

TABLE I

Value of the different Fisher tests on the transformed coefficient

The number of stars gives an indication of the value of $P$ for each $F$-test. When no star is indicated, the $F$-lest did not reach the level of significance of $P<0.05$. Usually, the interaction between the session number and the factor imager class was significant.

\begin{tabular}{lllll}
\hline Factors & $\begin{array}{l}\text { Catalogue } A \\
\text { 'picture-plane' } \\
\text { rotation }\end{array}$ & $\begin{array}{l}\text { Catalogue } A \\
\text { 'depth' rotation }\end{array}$ & $\begin{array}{l}\text { Catalogue B } \\
\text { 'picture-plane' } \\
\text { rotation }\end{array}$ & $\begin{array}{l}\text { Catalogue } B \\
\text { 'depth' rotation }\end{array}$ \\
\hline Session $(n=15)$ & $F_{14,73}=0.87$ & $F_{14,73}=1.91 *$ & $F_{14,72}=1.14$ & $F_{14,72}=1.39$ \\
Imagers $(n=2)$ & $F_{1,73}=0.17$ & $F_{1,73}=3.40$ & $F_{1,72}=1.10$ & $F_{1,72}=0.01$ \\
Session $\times$ Imagers & $F_{14,73}=2.44 * *$ & $F_{14,73}=1.48$ & $F_{14,72}=2.57 * * *$ & $F_{14,72}=2.58 * * *$ \\
\hline
\end{tabular}

${ }^{*} P<0.05,{ }^{* *} P<0.01,{ }^{* * *} P<0.005,{ }^{* * * *} P<0.001$. 
TABLE II

Value of the different F-tests on the intercept values

The number of stars gives an indication of the value of $P$ for each $F$-test. When no star is indicated, the $F$-test did not reach the level of significance of $P<0.05$. The interaction between the session number and the factor imaging skill never reached significance. Moreover, the session number alone usually did not reach significance, but the factor imaging skills always reached significance.

\begin{tabular}{llll}
\hline Factors & $\begin{array}{l}\text { Catalogue } A \\
\text { 'picture-plane' } \\
\text { rotation }\end{array}$ & $\begin{array}{l}\text { Catalogue } A \\
\text { 'depth' rotation }\end{array}$ & $\begin{array}{l}\text { Catalogue } B \\
\text { 'picture-plane' } \\
\text { rotation }\end{array}$ \\
\hline Session $(n=15)$ & $F_{14,73}=1.20$ & $F_{14,73}=1.41$ & $F_{14,72}=2.28 *$ \\
Imagers $(n=2)$ & $F_{1,73}=4.86 * 0+36^{*}$ & $F_{1,73}=5.29 *$ & $F_{1,72}=4.87 *$ \\
Session $\times$ Imagers & $F_{14,73}=0.56$ & $F_{14.73}=0.53$ & $F_{14,72}=0.43$ \\
\hline
\end{tabular}

${ }^{*} P<0.05, * * P<0.01, * * * P<0.005,{ }^{* * * *} P<0.001$.

plane' rotation $\left(F_{14,30}=1.3, P>0.2\right)$.

Over the training period, the 'good' imagers showed an improvement in the quality of the fit of the linear model during the first four sessions (reaching a maximum of 1.19 for 'depth' rotation), but decreased subsequently to a stable level (about 0.75 ) for the value of the transformed coefficient for catalogue $B$ and for both axes of rotation. For catalogue A, the effect was slightly different, there appeared first a decrease in the quality of the fit (reaching a minimum of 0.56 for 'depth' rotation), followed by an increase in the middle of the training period (reaching a maximum of 1.15 for 'depth' rotation). 'Picture-plane' rotation with stimuli from catalogue A tends to show similar effects as for 'depth' rotation (with the same catalogue) but usually did not reach significance. We supposed that this was due to a lesser complexity (see later) of both the type of rotation and of the catalogue (as compared to catalogue B).

In summary, the values of the transformed coefficient showed that the linear model is usually adequate to fit data for both groups, indicating that subjects mentally rotated stimuli as explained to them in the instructions. However, 'good' imagers seemed to be more likely to change strategies which they used to perform the task, than 'poor' imagers. This resulted in differences from session to session in the quality of the fit of the linear model.

\section{B - Intercept}

Imaging skill. A significant effect was obtained with the factor imaging skill (Table II). Usually, 'good' imagers required much less time to perform a $0^{\circ}$ difference in orientation judgement than 'poor' imagers (mean values for 'good' and 'poor' imagers, respectively: catalogue A, 'picture-plane' rotation $1.06 \mathrm{~s} / 1.51$ $\mathrm{s}$; catalogue $\mathrm{A}$, 'depth' rotation $1.07 \mathrm{~s} / 1.41 \mathrm{~s}$; catalogue $\mathrm{B}$, 'picture-plane' rotation $0.86 \mathrm{~s} / 1.19 \mathrm{~s}$; catalogue $\mathrm{B}$, 'depth' rotation $1.15 \mathrm{~s} / 1.56 \mathrm{~s}$ ).

Nevertheless, for each group and each axis of rotation, there was no significant difference in the time required for $0^{\circ}$ difference judgement $(t$-tests on catalogue A and B intercepts for 'good' and 'poor' imagers, respectively: 'picture-plane' rotation $t_{81}=2.5, P>$ $0.01 / t_{121}=1.73, P>0.05$; 'depth' rotation $t_{81}=0.86$, $P>0.35 / t_{121}=-0.95, P>0.3$ ).

Effect of training. The effect of the factor session number was generally not significant (see Table II), except for 'picture-plane' rotation for catalogue B. For this one exception, the effect of session number was only marginally significant $(P>0.01)$. This effect was contributed mainly by the first session, as the effect is

\section{TABLE III}

Value of the different $F$-tests on the slope of the regression lines

The number of stars gives an indication of the value of $P$ for each $F$-test. When no star is indicated, the $F$-test did not reach the level of significance of $P<0.05$. The interaction between the session number and the factor imaging skill never reached significance. However, each factors alone usually reached a level of significance.

\begin{tabular}{llll}
\hline Factors & $\begin{array}{l}\text { Catalogue } A \\
\text { 'picture-plane' } \\
\text { rotation }\end{array}$ & $\begin{array}{l}\text { Catalogue } A \\
\text { 'depth' rotation }\end{array}$ & $\begin{array}{l}\text { Catalogue B } \\
\text { picture-plane' } \\
\text { rotation }\end{array}$ \\
\hline Session $(n=15)$ & $F_{14,73}=3.77^{* * * *}$ & $F_{14,73}=2.69 * * *$ & $\begin{array}{l}\text { Catalogue } B \\
\text { 'depth' rotation }\end{array}$ \\
Imagers $(n=2)$ & $F_{1,73}=14.71^{* * * *}$ & $F_{1,73}=19.78^{* * * *}$ & $F_{1,72}=19.26^{*}$ \\
Session $\times$ Imagers & $F_{14,73}=0.67$ & $F_{14,73}=0.50$ & $F_{14.72}=2.5 * *$ \\
\hline
\end{tabular}

${ }^{*} P<0.05,{ }^{* *} P<0.01,{ }^{* * *} P<0.005,{ }^{* * * *} P<0.001$. 
no longer significant when the ANOVA is performed without the value of the first session. So, it seems that the training did not lessen substantially the time required to judge whether two figures in the same orientation are identical.

Imaging skill/training cross effect. There was no significant interaction (see Table II) between the two factors ( $P>0.85$ for the four $F$-tests).

In summary, there was no significant effect of training on the intercept, but an effect of imaging skill was demonstrated.

\section{$C$ - Slope of the regression lines}

Effect of training. There was a significant effect of session number on the slope of the regression lines: i.e. the mean slope decreased from session to session, or conversely, the presumed 'velocity' of mental rotation increased over the training period (see Table III).

When plotting the average value of slope for both catalogues against the number of session, as in Fig. 3 for 'picture-plane' rotation, the decrease in the slope for each catalogue can be summarised by fitting a decreasing exponential curve ( $r=0.92$ and $r=0.80$ for catalogues B and A, respectively). Similar effects appeared for 'depth' rotation.

A simple description of practice effect. Our aim was not to provide a highly reliable model of the mental rotation practice effect as a function of session number. The purpose underlying the use of an exponential curve to fit the data was only to provide a simple description of the data (with few parameters) so as to compare the effects of catalogues and imaging skills over the training period. A linear model was first

\section{"PICTURE-PLANE" ROTATION}

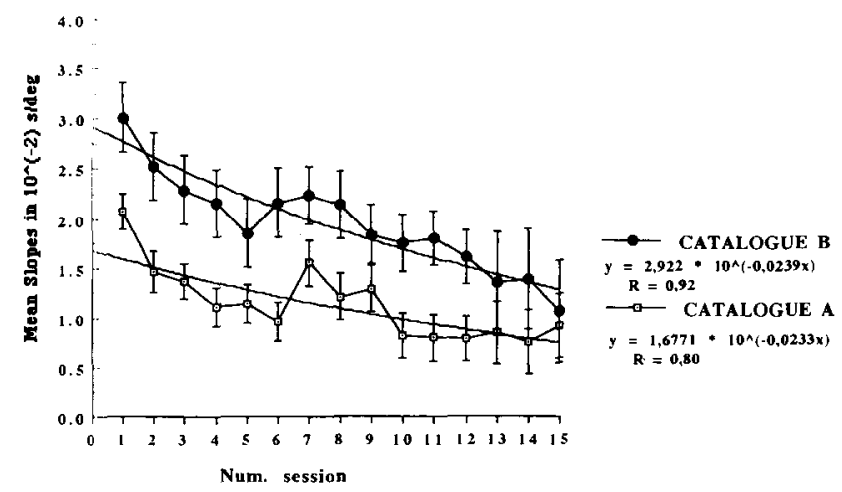

Fig. 3. Effect of the number of session on the slopes of the regression lines. For 'picture-plane' rotation and for each catalogue, we plotted the average slope (of all subjects tested on this catalogue) as a function of the session number. The black squares correspond to catalogue B and the white squares correspond to catalogue A. Bars indicate the standard deviation of each of the means. For each catalogue, an exponential curve, corresponding to the equation on the right was fitted to the data (see text).

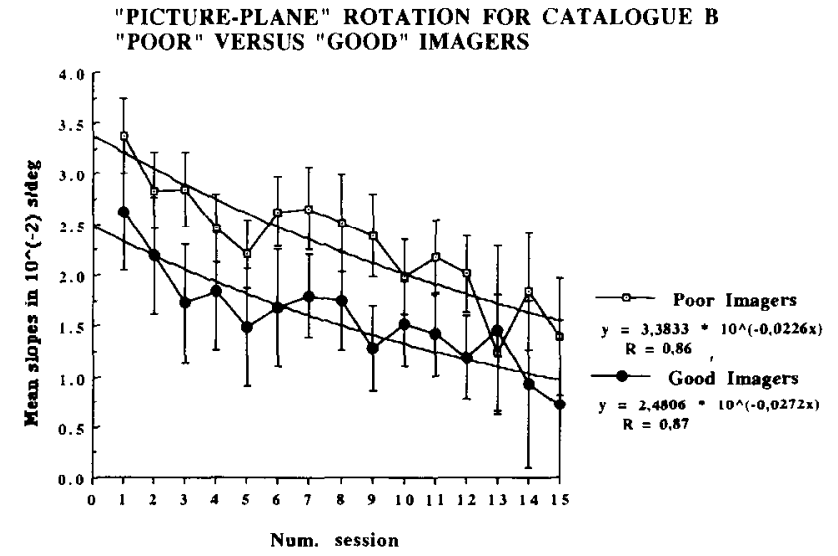

Fig. 4. Effect of session number on the slopes of the regression lines: difference between 'good' and 'poor' imagers. For 'picture-plane' rotation and for catalogue $\mathrm{B}$, we plotted the average slope (of all subjects tested on this catalogue and belonging to a given class of imaging skill) as a function of session number. The black squares correspond to the group of 'good' imagers and the white squares correspond to the group of 'poor' imagers. Error bars represent the standard deviation of the slopes. For each group, an exponential curve, corresponding to the equation on the right was fitted to the data.

tested, but the correlation coefficients between the linear model and the data were lower than for the exponential model.

The form of these exponential curves is: $S(n)=S(0)$ $\cdot 10^{(-G \cdot n)}$, where $n$ is the number of session, $S(n)$ is the computed value of the slope at session $n(V(n)=1$ $/ S(n)$ is the velocity of the mental rotation at session $n$ ) and $G$ is an adjusted parameter, representing the gain of the practice effect $\left(\log _{10}(S(n) / S(n+1))=\right.$ $\left.-\log _{10}(V(n) / V(n+1))\right)$ in the velocity of mental rotation from one session to the next. It appeared also that the gain $G$ was more or less independent of both the catalogue and the axis of rotation (mean \pm S.D.: 0.023 \pm 0.001 ). While the velocity increased for both catalogues, the initial difference between catalogue $A$ and $\mathrm{B}$ (that is the mean slope being higher for catalogue $\mathrm{B}$ than for catalogue $A$ by about $1.3 \cdot 10^{-2} \mathrm{~s} / \mathrm{deg}$ ) was maintained over the entire training period.

Imaging skill. A significant effect of imaging skill on the slopes of the regression lines also appears (see Table III). As shown in Fig. 4 for 'picture-plane' rotation and for catalogue $\mathrm{B}$, 'good' imagers had a higher velocity of mental rotation than 'poor' imagers. This difference in performance between 'good' and 'poor' imagers remained more or less constant over the training period. The same model of a decreasing exponential curve can be applied with good correlation $(r=0.87$ and $r=0.86$ for 'good' and 'poor' imagers, respectively) on each group of subjects.

Imaging skill/training cross effect. There was no significant interaction between the two factors $(P>$ 0.75 , for all of $4 F$-tests). 
Does 'mental rotation velocity' tend to infinity? We tested whether the rate of mental rotation reached an infinite value at the end of the training. As not all of our subjects were trained for 15 sessions, we decided to test the null hypothesis (i.e. that the rate of mental rotation is virtually infinite) at the 12 th session for all subjects. In fact, this null hypothesis is an unrealistic one, because it corresponds to an infinite velocity of the mental rotation. We therefore decided to test also the hypothesis that at the end of the training the velocity was higher than $300^{\circ}$ per second. This limit is subjective, but it seemed that with a computed velocity higher than this value, it would be difficult to report the use of a mental rotation process with our protocol, as the mean velocity of mental rotation reported by Shepard and Metzler ${ }^{27}$ was about $60^{\circ}$ per second and never excecded $300^{\circ}$ per sccond. Table IV reports the average values of the velocity at the 12 th session and the value of the one-tailed $t$-tests. All the $t$-tests showed a significant difference between the average velocity reached at the end of the training and the proposed value for the null hypothesis. Furthermore, the average velocities reached at the end of the training are within the range of values previously reported for such a paradigm $^{27}$.

Clearly, the rotation speed was always significantly lower than $300^{\circ}$ per second and never reached $13000^{\circ}$ per second as reported by Kaushall and Parsons ${ }^{16}$. These results support the idea that subjects used a mental rotation process during the entire training period.

\section{Effects of change of catalogues and novelty of stimuli}

From the previous results, it appears that, at least for the rate of mental rotation, performance on catalogue $B$ was not equal to performance on catalogue $A$. The mean velocity of mental rotation was higher for catalogue A (for both axis of rotation: $85^{\circ} / \mathrm{s}$ for 'picture-plane' rotation and $76^{\circ} / \mathrm{s}$ for 'depth' rotation) than for catalogue $\mathrm{B}\left(47^{\circ} / \mathrm{s}\right.$ and $52^{\circ} / \mathrm{s}$, respectively), the error rate was lower, but the intercept was not significantly different. In the following, we will say that the catalogue $\mathrm{B}$ is more complex than the catalogue $\mathrm{A}$ in the sense that: (1) the mean mental rotation velocity is higher for catalogue A than for catalogue B; and (2) subjects made fewer errors on catalogue $A$ than on catalogue B.

We decided to test the effect of the change of catalogue, mainly on the rate of mental rotation. Furthermore, the experimental design also allowed us to test for an effect of novel stimulus. Half of the subjects changed at session 7 from one catalogue to another which they had never seen before. The second half changed at the same time from one catalogue to another on which they had already trained during earlier practice sessions.

We will demonstrate in this part that the rate of mental rotation depends on the catalogue used, but does not depend on novelty of stimuli.

For each subject, we computed the difference in slopes (i.e. the inverse of rate of mental rotation) between the sixth and the seventh session. At this time, half of the subjects changed from catalogue $A$ to catalogue $\mathrm{B}$ (groups $3 \mathrm{~B} / 3 \mathrm{~A} / 3 \mathrm{~B} / 3 \mathrm{~A}$ and $6 \mathrm{~A} / 6 \mathrm{~B} / 3 \mathrm{~A}$ as explained in experimental design paragraph) and the other half changed from catalogue $B$ to catalogue $A$ (groups $3 \mathrm{~A} / 3 \mathrm{~B} / 3 \mathrm{~A} / 3 \mathrm{~B}$ and $6 \mathrm{~B} / 6 \mathrm{~A} / 3 \mathrm{~B}$ ).

We tested by a multifactor ANOVA: (i) the effect of change of catalogue (from $A$ to $B$ compared to from $B$ to A), (ii) the effect of novelty (subjects previously trained on a certain catalogue compared to subjects never trained on that catalogue) and (iii) the effect of imaging skills by an ANOVA on the difference on slope of mental rotation.

We separated in this analysis the two axes of rotation.

\section{Effect of change of catalogues}

For both types of rotation, there was a significant effect of change of catalogue $\left(F_{1.8}=12.44, P<0.01\right.$; $\left.F_{1,8}=12.35, P<0.01\right)$. This effect can be summarised

\section{TABLE IV}

\section{Value of the average telocity at the end of the training}

In the second and third rows, we tested whether rotation speed at the end of training reaches infinity or $300^{\circ}$ per second. The value of the $t$-tests as well as the significance level are given in these two rows.

\begin{tabular}{|c|c|c|c|c|}
\hline & $\begin{array}{l}\text { Catalogue } A \\
\text { 'picture-plane' } \\
\text { rotation }\end{array}$ & $\begin{array}{l}\text { Catalogue A } \\
\text { 'depth' rotation }\end{array}$ & $\begin{array}{l}\text { Catalogue B } \\
\text { 'picture-plane' } \\
\text { rotation }\end{array}$ & $\begin{array}{l}\text { Catalogue } B \\
\text { 'depth' rotation }\end{array}$ \\
\hline $\begin{array}{l}\text { Average velocity } \\
\text { Ho }(1 / V=0) \\
\text { H3 }(1 / V=1 / 300)\end{array}$ & $\begin{array}{l}125^{\circ} / \mathrm{s} \\
t_{7}=4.72 * * * \\
t_{7}=2.76 *\end{array}$ & $\begin{array}{l}99^{\circ} / \mathrm{s} \\
t_{7}=3.14 * * \\
t_{7}=2.11^{*}\end{array}$ & $\begin{array}{l}61^{\circ} / \mathrm{s} \\
t_{9}=6.9 * * * * \\
t_{9}=5.52 * * * *\end{array}$ & $\begin{array}{l}71^{\circ} / \mathrm{s} \\
t_{9}=4.9 * * * * \\
t_{9}=3.75 * * *\end{array}$ \\
\hline
\end{tabular}

${ }^{*} P<0.05,{ }^{* *} P<0.01,{ }^{* * *} P<0.005,{ }^{* * * *} P<0.001$. 
TABLE V

Value of the difference in slopes (in $10^{-2} \mathrm{~s} / \mathrm{deg}$ ) between two consecutive sessions

Some subjects changed from one catalogue to another (transitions A-B and B-A) or not (transitions A-A and B-B). For each axis of rotation and for 3 critical sessions in the training (session 3,6 and 9), we computed the average difference in slope with the following session as well as the standard deviation.

\begin{tabular}{llrrrrr}
\hline Transitions & $\begin{array}{l}\text { 3th-4th } \\
\text { 'picture- } \\
\text { plane' rot. }\end{array}$ & $\begin{array}{l}\text { 3th-4th } \\
\text { 'depth' rot. }\end{array}$ & $\begin{array}{l}\text { 6th-7th } \\
\text { 'picture- } \\
\text { plane' rot. }\end{array}$ & $\begin{array}{l}\text { 6th-7th } \\
\text { 'depth' rot. }\end{array}$ & $\begin{array}{l}\text { 9th-10th } \\
\text { 'picture- } \\
\text { plane' rot. }\end{array}$ & $\begin{array}{l}9 \text { th-10th } \\
\text { 'depth' rot. }\end{array}$ \\
\hline A-B & $-0.69 \pm 0.58$ & $-0.75 \pm 0.47$ & $-1.26 \pm 0.41$ & $-0.81 \pm 0.32$ & $-0.47 \pm 0.42$ & $-0.36 \pm 0.38$ \\
B-A & $1.12 \pm 1.78$ & $0.41 \pm 0.92$ & $0.73 \pm 0.96$ & $0.66 \pm 0.81$ & $0.54 \pm 0.40$ & $1.10 \pm 0.69$ \\
A-A & $0.32 \pm 0.07$ & $0.51 \pm 0.22$ & & & $0.45 \pm 0.84$ & $0.49 \pm 1.30$ \\
B-B & $0.28 \pm 0.54$ & $-0.01 \pm 0.70$ & & $0.07 \pm 0.39$ & $0.01 \pm 0.13$ & \\
\hline
\end{tabular}

by the fact that when the transition was from $\mathrm{A}$ to $\mathrm{B}$, subjects showed a decrease in the slope of mental rotation (average change of slopes of $-1.26 \cdot 10^{-2}$ $\mathrm{s} / \mathrm{deg}$ and $-0.81 \cdot 10^{-2} \mathrm{~s} / \mathrm{deg}$ for 'picture-plane' and 'depth' rotation), but conversely when the transition was from $B$ to $A$, subjects showed an increase in the rate of mental rotation (average change of slopes of $0.73 \cdot 10^{-2} \mathrm{~s} / \mathrm{deg}$ and $0.66 \cdot 10^{-2} \mathrm{~s} / \mathrm{deg}$ for 'pictureplane' and 'depth' rotation).

Furthermore, this transition effect can be shown at other periods of the training as well. Table $\mathrm{V}$ gives the average differences in the inverse of the rate of mental
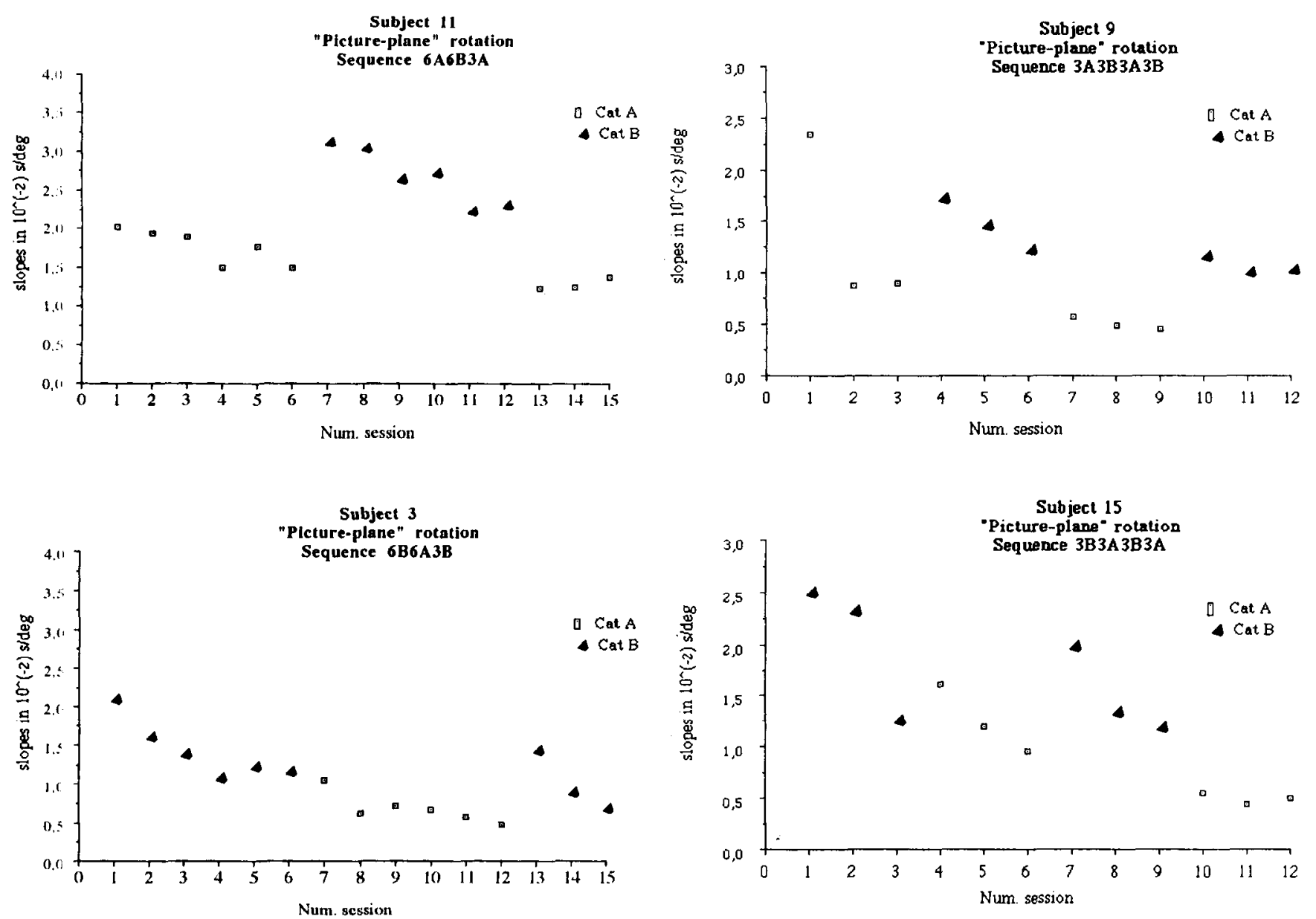

Fig. 5. Effect of the change of catalogue during the training for four subjects, one from each experimental group. We plotted the slope of the regression line for 'picture-plane' rotation as a function of the session number. Each subject was trained on both catalogues in different sequences. For each subject, we notice the general effect of training (decrease in the rate of mental rotation) as well as the effect of complexity of catalogue, neither of which disappeared over the training period. 
rotation between sessions $n$ and $n+1$, as well as the standard deviation, when $n$ corresponds to a change of catalogue.

It appears that a change in training from catalogue A to catalogue $B$ always entailed a decrease in the mental rotation velocity used with catalogue $B$ compared to the one used with catalogue $\mathrm{A}$, independent of the point in time at which the transition occurred during the training period. The change from $\mathrm{B}$ to $\mathrm{A}$ entailed always a strong increase in the rate of mental rotation.

\section{No novelty effect}

There were no significant interactions of second or third order between these three factors (the change of catalogue, the novelty effect and the imaging skill) ( $P>0.35$ for the eight F-tests). There was no effect of imaging skills alone $\left(F_{1,8}=0.014, P>0.9 ; F_{1,8}=0.20\right.$, $P>0.65$ for 'picture-plane' and 'depth' rotation, respectively $)$ or of novelty alone $\left(F_{1.8}=1.32, P>0.25\right.$; $F_{1.8}=1.17, P>0.30$ ).

In summary, subjects showed a clear influence of practice, but this influence of practice seemed dependent on the complexity of the catalogue used at a given session. This effect of complexity did not disappear over the training period and was not related to an effect of novelty of stimuli.

Fig. 5 shows the effect of change of catalogues on inverse of rate of mental rotation for four different subjects belonging to each of the four groups of our experimental design. The effect reported previously for the overall behavior of each group shows up clearly for individual responses as well.

Does the training with one catalogue extend to the other? As the effect of complexity of stimuli seems to be important, we can ask if there was a kind of procedural learning over the training. For example, if a subject was trained on catalogue $A$, which appears to be less complex, will he or she show an improvement of the mental rotation rate when trained on catalogue $B$, as compared to a subject trained directly on catalogue B? To test this hypothesis, we compared the mean inverse of the rates of mental rotation (over three consecutive sessions in order to reduce the variability of the first session) obtained during the three first sessions for subjects beginning experiments with catalogue $\mathrm{B}$ (groups $3 \mathrm{~B} / 3 \mathrm{~A} / 3 \mathrm{~B} / 3 \mathrm{~A}$ and $6 \mathrm{~B} / 6 \mathrm{~A} / 3 \mathrm{~B}$ ), with the mean inverse of the rates of mental rotation obtained for the fourth, fifth, and sixth or the seventh, eighth, and ninth sessions with subjects already trained on catalogue $A$ (groups $3 A / 3 B / 3 A / 3 B$ and $6 A / 6 B /$ $3 \mathrm{~A}$, respectively).

The results (see Table VI) showed a trend towards a
TABLE VI

Value of the average slope (in $10^{-2} \mathrm{~s} / \mathrm{deg}$ ) for 3 consecutive sessions Group 0 is constituted of subjects beginning the training with the specified catalogue, noted at the head of the column (A or B). Group 1 represents subjects trained with the other catalogue during the 3 first sessions then trained with the specified catalogue during session 4 to 6 . Group 2 represents subjects trained with the complementary catalogue during the six first sessions then trained with the specified catalogue during session 7 to 9 .

\begin{tabular}{lllll}
\hline $\begin{array}{l}\text { Catalogue } A \\
\text { 'picture-plane' } \\
\text { rotation }\end{array}$ & $\begin{array}{l}\text { Catalogue } A \\
\text { 'depth' } \\
\text { rotation }\end{array}$ & $\begin{array}{l}\text { Catalogue } B \\
\text { 'picture-plane' } \\
\text { rotation }\end{array}$ & $\begin{array}{l}\text { Catalogue } B \\
\text { 'depth' } \\
\text { rotation }\end{array}$ \\
\hline Group 0 & $1.80 \pm 0.51$ & $1.71 \pm 0.52$ & $2.51 \pm 0.62$ & $2.77 \pm 1.14$ \\
Group 1 & $1.31 \pm 0.44$ & $1.69 \pm 0.79$ & $2.17 \pm 0.76$ & $2.33 \pm 0.77$ \\
Group 2 & $1.77 \pm 1.24$ & $1.88 \pm 1.05$ & $1.50 \pm 0.75$ & $1.97 \pm 0.67$ \\
\hline
\end{tabular}

higher rate of mental rotation obtained for subjects already trained on this task with another catalogue. Nevertheless, this difference was never statistically significant, perhaps due to the small sample size.

It seemed also that subjects trained with a complex catalogue (catalogue $\mathrm{B}$ ), then changed to a simpler one (groups $3 \mathrm{~B} / 3 \mathrm{~A} / 3 \mathrm{~B} / 3 \mathrm{~A}$ and $6 \mathrm{~B} / 6 \mathrm{~A} / 3 \mathrm{~B}$ ), showed similar results as subjects beginning the task with the simple one. Yet subjects trained with a simple catalogue tended to show better performance when they changed to a complex catalogue than subjects beginning the training with the complex catalogue. Moreover, a slight improvement appeared when subjects were trained during 6 sessions (as compared to 3 ) with the easiest catalogue, prior to the change in the complexity of catalogue.

\section{Subjects' reports}

In the following paragraph, we report some spontaneous remarks made by some subjects at the end of the sessions. Most of the subjects reported that they mentally rotated the right image and noticed that 'pictureplane' rotations seemed easier. A specific rotation sense (clockwise versus counter clockwise) was difficult to define for them. Two subjects mentally rotated themselves around the objects, but only until the third to the fifth session. Some tried to manipulate mentally the left perspective or to rotate themselves mentally but they explained that such strategies led to difficulties in the correct achievement of the task.

With practice, mental rotation was said to be much more easily applied and some refinements in strategies occurred. Some of the subjects referred to a double rotation: i.e. rotations of both views during intermediate sessions, and one subject had the feeling of rotating himself and the ohject.

The subjects became aware of the introduction of new shapes but they were not sure that the whole set 
had changed. All had the feeling of 'having already seen' shapes after three consecutive sessions and the feeling of achieving sometimes a 'direct identification', without the need for the mental rotation process. Some subjects clearly indicated a memorisation occurring 'by pair'.

The feeling of complexity was explained by the number of cubes in the ends of the 3-D structures. They seemed to use both holistic or sequential segment by segment rotations of objects. No specific strategy was associated which to either the gender of the subject, or the ability to perform the task, or the catalogue. Some subjects indicated that they perceived objects' discriminative parts ('arms' and 'joints'), but they did not feel that they performed the mental rotation of these parts only. However, perceiving such distinctive parts could trigger the mental rotation of the whole representation of an object.

\section{DISCUSSION}

\section{Influence of practice}

Our main results concerning the influence of longterm practice on mental rotation may be summarized as follows:

(i) We observed a linear relation between response times and angular differences for all sessions and all subjects.

(ii) The inverse of the rate of mental rotation decreased as a function of session number and this decrease was adequately fitted by an exponential curve.

(iii) We have shown a dependence of mental rotation velocity on both the set of objects used and on the intrinsic imaging skills of the subjects (as measured by the paper Mental Rotation Test ${ }^{33}$ ).

(iv) The gain in mental rotation velocity from one session to the next did not depend on either the catalogue of images or the imaging skill of the subject: the initial difference in performance due to either differences in the catalogues or differences in imaging skill remained constant over the training period.

(vi) Being trained on an easier catalogue seems to facilitate the acquisition of mental rotation skills.

(vii) Under our experimental conditions (large set of stimuli and long-term practice), the training did not lead to the disappearance of the mental rotation process.

Our results differ from those of previous studies as mentioned in the introduction: i.e. we saw neither the disappearance of the linear relationship between reac- tion time (RT) and orientation (OD) as observed by Kaushall and Parsons ${ }^{16}$ nor the disappearance of the complexity dependence or the novelty effect as seen in Bethell-Fox and Shepard's experiment ${ }^{3}$. These discrepancies do not correspond to contradictory theories but rather represent differences with regard to experimental conditions. Kail and Park ${ }^{15}$ propose an interesting theory for the general mechanisms occurring with the practice of mental rotation. According to this theory, practice increases the strength and the number of representations of a given object stored in memory. For the first trials, only a few representations are available and the response times are thus much more likely to correspond to the execution of the mental rotation algorithm. With practice, the response to a well-learned stimulus is instead retrieved directly from memory.

Therefore, the identification tasks involve both a mental rotation process in itsclf and a memory retrieval process. Practice influences the mental rotation process as well as the acquisition of significant features in the encoding space. These two components are simultaneously engaged but the predominant process (mental rotation vs memory retrieval), as revealed by the response times, depends on the degree of storage of stimuli. In the limit, this theory would predict that mental rotation would disappear with practice. All objects orientation would eventually be stored, and retrieved directly from memory.

This hypothesis is purely theoretical but provides an explanation for: the independence of response time from angle in the Kaushall and Parsons's experiment ${ }^{16}$ that may be attributed to the phenomenon of memorisation; the number of sessions in the Bethell-Fox and Shepard's experiment ${ }^{3}$ that enables one to compensate for the complexity. The novelty effect may be due to the occurrence of new features, though the algorithm of equality judgement is not yet computed optimally.

Since memorisation was not easy in the present experiment, the mental rotation process is still preponderant by the last session. However, according to comments made by subjects, some responses seem to be directly retrieved.

It is worth noticing that these two components do not seem to be completely independent, i.e. the identification task would be facilitated when working on data easily encoding.

Well acquired internal representation could lead to a velocity which is independent of the complexity, as argued by many authors ${ }^{3,7,8}$, but our training may not have been sufficiently long to see this result. The similarity (gain and shape) of the two lcarning curves for both catalogues reinforces the assumption that it is 
an unique mental rotation process which is available and that it persists over the training period.

Nevertheless, we will propose a new interpretation of mental rotation that explains differently this observation (see below).

\section{Individual imaging ability}

The main results concerning individual imaging ability according to the two classes that we defined are:

(i) the error rate is higher for the catalogue B for the two classes, but 'good' imagers made fewer errors on both catalogues over the entire training period.

(ii) The mental rotation slope is lower for 'good' imagers, i.e the mental rotation velocity was higher, and no speed/accuracy trade-off was observed for either group.

(iii) There is no significant effect of session number on the quality of the fit of the linear model for 'poor' imagers, though there is a significant effect for 'good' imagers.

(iv) The intercept is lower for 'good' imagers.

As mentioned in the introduction, individual ability to perform mental rotation tasks may be attributed to general aptitudes in spatial viewing. Kosslyn and al. ${ }^{18}$ and Goldston et al. ${ }^{11}$ found individual differences in a variety of imagery tasks. Lohman ${ }^{20}$ postulated that high spatial ability implies a better accuracy in solving complex rotation problems.

Just and Carpenter ${ }^{14}$ studied the preferred cognitive coordinate system used by subjects. 'Poor' imagers seem to use cognitive coordinate systems that are near the standard axes of the stimuli or environment. They proposed that rotations are made by sequential steps of rotation but theses steps are larger for 'good' imagers. In addition, they argued that the former have poor book-keeping forces that oblige them to do subsequent rotation and confirmation. 'Good' imagers manipulate much more easily than 'poor' imagers objects that remain structured and some studies also support the idea that they generate images more quickly than 'poor' imagers ${ }^{6,10}$. As noted above, this easy manipulation enables them to use more flexible strategies (significant effect of session number for 'good' imagers).

However, these differences may reveal different ways of processing mental images. Steiger and Yuille ${ }^{30}$ noted individual differences in the 'standard' orientation of a stored stimulus to explain their results observed under conditions of memorisation. Moreover, some experiments have shown that imagers exhibit variable capacities in each modulus of the human mental imagery treatment $^{18}$.
These considerations and the fact that, in our experiment, the error rate and the intercept are greater for 'poor' imagers, show that these latter have probably more difficulties to extract and code features of 3-D objects. The intercept is commonly referred to the identification time at $0^{\circ}$. In our experiment, the gain in mental rotation velocity from one session to the next did not depend on imaging skill.

\section{Catalogue effect}

Our results demonstrated differences between the two catalogues of test images. When performing the mental rotation task with catalogue $\mathrm{B}$, all subjects, whatever their imaging skill, gave more incorrect responses and showed a lower 'velocity' of mental rotation than which catalogue $\Lambda$. It was also demonstrated that these differences did not result from a novelty effect of stimuli, and did not disappear over the training period. That last remark runs against previous results obtained by Bethell-Fox and Shepard ${ }^{3}$. They found that the initial 'velocity' of mental rotation was dependent on the complexity of stimulus, but this dependence disappeared with practice. Nevertheless, the velocity of mental rotation depended on the novelty of stimulus. Thus, a novel stimulus with a lower complexity than a well learned stimulus, was processed at a lower rate of mental rotation. Cooper and Podgorny ${ }^{8}$ found that the velocity of mental rotation was independent of the complexity of 2-D polygons (as counted by the number of sides), but they did not specify the level of practice of the subjects.

These previous results ${ }^{3,8}$ were achieved with a different protocol than ours. First, they involved 2-D stimuli (matrices or polygons). Second, the task relied on a 'memorised' mental rotation paradigm, in the sense that subjects had to judge whether the displayed stimulus is identical to a previously memorised stimulus. Third, the intersession rest time was short, as the entire experiments lasted less than $3 \mathrm{~h}$ (compared to approximately 1.5 month, for our experiment). We will attempt to see whether our results can be attributed to one of these differences.

One of the advantages of using 2-D stimuli in a mental rotation task was the number of studies made to define a measure of complexity for such figures ${ }^{1,2}$. Using such a measure of complexity for 2-D polygons, based on the number of sides, Cooper ${ }^{7}$ found that the rate of mental rotation was independent of the measured complexity of stimuli. However, Yuille and Steiger ${ }^{34}$ pointed out that the measure of complexity used by Cooper and Podgorny ${ }^{8}$ is not an effective measure of complexity for the mental rotation task, as 
'the more complex forms contain distinguishing featurcs so that the entire figure does not have to be examined when comparing it with a second figure'.

Nevertheless, the stimuli used in our experiment are perspective views of 3-D objects. Some studies have been done to give a measure of complexity with drawings of 3-D objects. Butler ${ }^{5}$ proposed a measure of complexity of drawings of wire frame objects, as well as, of solid objects, based on previous works ${ }^{4,12}$, in which 'the complexity is measured by adding the information load computed using coding theory and the number of lines'. This measure seems effective for predicting the perceived dimensionality of objects drawn, as a function of measured complexity, but this measure is very difficult to perform on our stimuli, and moreover it remains dependent on the point of view from where the object is seen.

In fact, our results have shown that the velocity of mental rotation is similar for 'picture-plane' and 'depth' rotation for a given catalogue. As hypothesized already ${ }^{27}$, our results seem to indicate that the subjects had internal 3-D representations of objects, and that measures of complexity based on 2-D drawings are not adequate. The results of Kaushall and Parsons ${ }^{16}$ lead to similar conclusions as they have shown that there was no facilitative effect of having viewed the objects from various perspectives. The task remains to find a measure of complexity based on the 3-D structure of objects which can explain the differences in the mental rotation obtained. Shepard and Metzler ${ }^{26}$ proposed that the number of cubes in an object which they used as stimulus, could be a valuable measure of complexity. Yuille and Steiger ${ }^{34}$ proposed a similar measure based on the number of cubes as long as there is no feature redundance in the figure. These authors pointed out that the more complex the 3-D object was, the slower the rate at which subjects mentally rotated it. However, subjects can sometimes use a strategy exploiting 'figural redundancies' of objects to perform mental rotation. From their point of view, the mental rotation task showed a clear practice effect, and consequently, as argued by Pylyshyn ${ }^{23}$, the process of mental rotation is 'cognitively penetrable'. Thus, the complexity dependence of the process was a key argument for rejecting Shepard and Metzler's hypothesis ${ }^{27}$. This hypothesis stipulates that mental rotation is an 'holistic' or 'analogue' process in the sense that subjects mentally rotate the 'whole' internal representation of the stimulus.

On the opposite, Yuille and Steiger ${ }^{34}$ supposed that 'the mental rotation task is performed by a series of sequential comparisons of figure segments'. In this 'piecemeal' interpretation of mental rotation, they supposed that subjects first searched for similar segments in the two views (usually the 'arms' of the figures), then compared them and if there were identical, computed and stored information about the angular discrepancy between these two segments. This process would be repeated for subsequent segments. They noticed that this hypothesis finds some support in the study of eye movements during a mental rotation task ${ }^{13}$.

\section{New hypothesis on mental rotation}

Taking into account our results, as well as previous results $^{22,33}$, we propose a new interpretation of the process of mental rotation. The key point in our hypothesis is that, during an identity judgement of 3-D objects, subject mentally rotates an object-centered reference frame, called in the following the 'principal plane', and not a internal representation of an entire 3-D object or an internal representations of subsequent segments of 3-D objects.

More specifically, we suppose that subjects will initially define what we call the 'principal' plane of the 3-D object. With Shepard's stimuli (ten contiguous cubes, four branches and three right angles), this 'principal' plane is defined as the plane containing the two middle branches. We note that generally in our experiment, the plane containing the central joint is also the plane containing the maximal number of contiguous cubes. By extension to other assemblies of contiguous cubes ${ }^{34}$, the 'principal' plane is defined as the plane on which the maximal number of contiguous cubes of the object lies.

With our stimuli, the 'principal' plane contained from 5 to 9 , out of the ten possible cubes (see Fig. 2). Our main hypothesis is that subjects mentally rotate in a 'holistic' way the internal representation of the part of the object belonging to this 'principal' plane, and not the entire representation of the object. All the cubes out of this 'principal' plane would be processed separately in a kind of 'search and confirmation' strategy (see ref. 13 for the terminology). Thus, the greater the number of cubes out of this 'principal' plane, the more different features one has to manipulate, and the greater the amount of time required to execute the mental manipulation. We can also imagine that the number of cubes out of this plane is not so important as compared to the number of segments perpendicular to this 'principal' plane. Further experiments should resolve this point. Nevertheless, if our assumption is correct, previous experiments would give no evidence that the mental rotation of what is actually rotated (the 'principal' plane) is a complexity-dependent process.

With our interpretation of mental rotation, the number of cubes out of this 'principal' plane would 
predict the differences in mental rotation rate between objects. The more numerous the outlying cubes are, the slower the velocity of mental rotation. Thus, with catalogue $\mathrm{A}$, there are on average 1.83 cubes out of the objects' 'principal' plane (range from 1 to 3 ), but with the catalogue $B$, there are on average 3.5 cubes out (range from 2 to 5). We suppose that this difference in the number of out-of-plane cubes explains the highly significant difference in the rate of mental rotation between catalogues $\mathrm{A}$ and $\mathrm{B}$.

Similarly, such a hypothesis can explain the results of the experiment of Yuille and Steiger ${ }^{34}$ in which the so-called 'twisted' objects are processed more slowly than 'non-twisted' objects. In fact, for the 'twisted' objects, the number of cubes out of object's 'principal' plane was bigger than in the case of 'non-twisted' objects. We suppose that this is the reason why the rate of mental rotation of 'twisted' objects is slower than the rate of mental rotation for 'non-twisted' objects. Moreover, we can explain by our intepretation the results of Metzler and Shepard ${ }^{22}$. These authors have shown a difference in the mental rotation velocity between similar 3-D objects. They explained that the lower rate of mental rotation of object designated $\mathrm{C}$ in the paper ${ }^{26}$ was due to the presence of almost symmetrical features in this object, which was not the case for the four others. In fact, it is also clear that this object has four cubes out of its 'principal' plane while the four other objects had all only two cubes out of their 'principal' plane. Without denying that some structural properties of objects could help or hinder the mental rotation process, we can explain Shepard's results by our model.

In conclusion, we propose a new interpretation of mental rotation based on the extraction by the subject of a structural variable of objects, their 'principal' plane, by the subject. We suggest that the subject mentally rotates the internal representation of the object's part lying in this plane and not the whole object's representation ('holistic' point of view) or segment by segment ('piecemeal' point of view). The existence of cubes which lie out of this 'principal' plane will decrease the velocity of mental rotation. Though this model must be clarified by further experiments, our intepretation can already resolve previous contradictory results.

In this study, we demonstrate that the subjects still use a strategy of mental rotation over long-term practice with an appropriate number of stimuli. The influence of practice on mental rotation of 3-D objects results mainly in an improvement of velocity of mental rotation. In addition, we show that initial differences in mental rotation performances, due to spatial abilities of subjects or complexity of 3-D objects used, do not disappear over the training period.

Acknowledgements. We thank Yoanni Matsakis (Institut de Médicine spatiale), Olivier Charade (Laboratoire de Physiologie de la Perception et de l'Action) and A.E.T.A for technical support. We acknowledge Michel Denis for preliminary discussion about this work. We are indebted to Alain Berthoz (Laboratoire de Physiologie de Perception el de l'Actioni) and Marc Lipshits (Institute for Problems of Information transmission) for comments on the manuscript. We particularly acknowledge Joe Mc Intyre (Laboratoire de Physiologie de Perception et de l'Action) for his helpful comments and rewriting work. This research was in part supported by a grant from C.N.E.S (Centre National d'Etudes Spatiales) $(92 / \mathrm{CNES} / 0247)$ and a grant from Ecole Polytechnique (DRET 91-815/10).

\section{REFERENCES}

1 Attneave, F., Physical determinants of the judged complexity of shapes, J. Exp. Psychol., 53 (1957) 221-227.

2 Attneave, F. and Arnoult, M.D., The quantitative study of shape and pattern perception, Psychol. Bull., 53 (1956) 452-471.

3 Bethell-Fox, C.E and Shepard, R.N., Mental rotation: effect of stimulus complexity and familiarity, J. Exp. Psychol.: Human Perc. Perf., 14 (1988) 12-23.

4 Buffart, H., Leeuwenberg, E. and Restle, F., Coding theory of visual pattern completion, J. Exp. Psychol.: Human Perc. Perf., 7 (1981) 241-274.

5 Butler, D.L., Predicting the perception of three dimensional objects from the geometrical information in drawings, J. Exp. Psychol.: Human Perc. Perf., 8 (1982) 674-692.

6 Cocude, M. and Denis, M., Measuring the temporal characteristics of visual images, J. Mental Imagery, 12 (1988) 89-101.

7 Cooper, L.A., Mental transformation of random 2D shapes, Cogn. Psychol., 7 (1975) 20-43.

8 Cooper, L.A. and Podgorny, P., Mental transformations and visual comparison processes: effects of complexity and similarity, J. Exp. Psychol:: Human Perc. Perf., 2 (1976) 503-514.

9 Denis, M., Approches différentielles de l'imagerie mentale. In M Reuchlin, J. Lautrey, C. Marendaz and T. Ohlman (Eds.), Cognition: l'individuel et l'unicersel, Presses Universitaires de France, Paris, 1990, pp. 91-120.

10 Ernest, C.H. and Paivio, A., Imagery and verbal associative latencies as a function of imagery ability, Can. J. Psychol., 25 (1971) 83-90.

11 Goldston, D.B., Hinrichs, J.V. and Richman, C.L., Subject's expectations, individual variability, and the scanning of mental images, Mem. Cogn., 13 (1985) 365-370.

12 Hochberg, J.E. and Brooks, V., The psychophysics of form: reversible perspective drawings of spatial objects, Am. I. Psychol, 73 (1960) 337-354.

13 Just, M.A. and Carpenter, P.A., Eye fixations and cognitive processes, Cogn. Psychol., 8 (1976) 441-480.

14 Just, M.A. and Carpenter, P.A., Cognitive coordinate systems: accounts of mental rotation and individual differences in spatial ability, Psychol. Rev., 92 (1985) 137-171.

15 Kail, R. and Park, Y.S., Impact of practice on speed of mental rotation, J. Exp. Child Psychol., 49 (1990) 227-244.

16 Kaushall, P. and Parsons, L.M., Optical information and practice in the discrimination of $3 \mathrm{D}$ mirror-reflected objects, Perception, 10 (1981) 545-562.

17 Kosslyn, S.M., Image and Mind, Harvard University Press, Cambridge, MA, 1980.

18 Kusslyn, S.M., Brunn, M., Cave, K. and Wallach, R.W., Individual differences in mental imagery: a computational analysis, $\mathrm{Cog}$ nition, 18 (1984) 195-243.

19 Kosslyn, S.M. and Jolicoeur, P., A theory-based approach to the study of individual differences in mental imagery. In R.E. Snow, P.A. Federico and W.E. Montague (Eds.), Aptitude, Learning and 
Instruction, vol 2: Cognitice Process analysis of Learning and Problem Solving, Lawrence Erlbaum Associated, Hillsdale, NJ, 1982 .

20 Lohman, D.F., The effect of speed accuracy tradeoff on sex dilferences in mental rotation, Percept. Psychophys., 36 (1986) $427-436$.

21 Metzler, J., Cognitive Analogue of the Rotation of 3-D Objects, 1973, unpublished.

22 Metzler, J. and Shepard, R.N., Transformational studies of the internal representation of three dimensional objects. In R. Solso (Ed.), Theories in Cognitive Psychology: The Loyola Symposium, Lawrence Erlbaum Associated, Hillsdale, NJ, 1974.

23 Pylyshyn, Z.W., The rate of mental rotation of images: a test of holistic analogue hypothesis, Mem. Cogn., 7 (1979) 19-28.

24 Richardson, J.T.E., Gender Differences in Mental Rotation, Proce'dings of the Fourth European Workshop on Imagery and Cognition, Tenerife, Spain, 1992.

25 Schwartz, S.P. and Kosslyn, S.M., A computer simulation approach to studying mental imagery. In J. Mehler, E.C.T. Walker and M. Garrett (Eds.), Perspectives on Mental Representation: Experimental and Theoretical Studies of Cognitive Process and Capacities, Lawrence Erlbaum Associated, Hillsdale, NJ, 1982, pp. 69-85.
26 Shepard, R.N. and Cooper, L.A., Mental Images and their Transformations, MIT Press, MA, 1982.

27 Shepard, R.N. and Metzler, J., Mental rotation of three dimensional objects, Science, 171 (1971) 701-703.

28 Snedecor, G.W. and Cochran, W.G., Statistical Methods, 8th edn., Iowa State Press University, 1989.

29 Steel, R.G.D. and Torrie, J.H., Principles and Procedures of Statistics, McGraw-Hill, New York, 1960

30 Steiger, J.H. and Yuille, J.C., Long-term memory and mental rotation, Can. J. Psychol, 37 (1983) 367-389.

31 Taine, M.C., Leone, G., Berthoz, A. and Droulez, J., Practice effect on mental rotation of 3D objects, Proceedings of the Fourth European Workshop on Imagery and Cognition, Tenerife, Spain, 1992.

32 Tapley, S.M. and Briden, M.P., Sex differences in spatial ability: mental rotation of three dimensional objects, Can. J. Psychol., 31 (1977) $122-130$.

33 Vandenberg, S.G. and Kuse, A.R., Mental rotation, a group test of three dimensional spatial visualization, Percept. Motor Skills, 47 (1978) 599-604.

34 Yuille, J.C. and Stcigcr, J.H., Nonholistic processing in mental rotation: some suggestive evidence, Percept. Psychophys., 31 (1982) 201-209. 